arXiv version: fonts, pagination and layout may vary from AGT published version

\title{
Cohomology of Coxeter groups with group ring coefficients: II
}

\author{
MiCHAEL W DAVIS \\ JAN DYMARA \\ TADEUSZ JANUSZKIEWICZ \\ BORIS OKUN
}

\begin{abstract}
For any Coxeter group $W$, we define a filtration of $H^{*}(W ; \mathbf{Z} W)$ by $W$-submodules and then compute the associated graded terms. More generally, if $\mathcal{U}$ is a $\mathrm{CW}$ complex on which $W$ acts as a reflection group we compute the associated graded terms for $H_{*}(\mathcal{U})$ and, in the case where the action is proper and cocompact, for $H_{c}^{*}(\mathcal{U})$.
\end{abstract}

20F55; 20C08, 20E42, 20F65, 20J06, 57M07

\section{Introduction}

The cohomology of a group $G$ with coefficients in a left $G$-module $M$ is denoted $H^{*}(G ; M)$. We are primarily interested in the case where $M$ is the group ring, $\mathbf{Z} G$. Since $\mathbf{Z} G$ is a $G$-bimodule, $H^{*}(G ; \mathbf{Z} G)$ inherits the structure of a right $G$-module. When $G$ is discrete and acts properly and cocompactly on a contractible $\mathrm{CW}$ complex $\Omega$, there is a natural topological interpretation for this cohomology group: $H^{*}(G ; \mathbf{Z} G) \cong H_{c}^{*}(\Omega)$, where $H_{c}^{*}($ ) denotes finitely supported cellular cohomology. The action of $G$ on $\Omega$ induces a right action on cohomology and the above isomorphism is one of right $G-$ modules. For a general group $G$, not much is known about the $G$-module structure on $H^{*}(G ; \mathbf{Z} G)$. For example, even in the above case where $G$ acts properly and cocompactly on a contractible $\Omega$, we don't believe it is known whether or not $H^{*}(G ; \mathbf{Z} G)$ is always finitely generated as a $G$-module.

Here we deal with the case where $G=W$, a Coxeter group. In [5] the first author computed $H^{*}(W ; \mathbf{Z} W)$ as an abelian group but not as a $W$-module. We partially remedy the situation here. We do not quite determine the $W$-module structure on $H^{*}(W ; \mathbf{Z} W)$. Rather, we describe a certain decreasing filtration of $H^{*}(W ; \mathbf{Z} W)$ by $W$-submodules 
and compute the associated graded terms. In order to describe this computation, we need some notation.

Suppose $(W, S)$ is a Coxeter system. ( $W$ is the group and $S$ is the distinguished set of involutions which generates $W$.) A subset $T \subset S$ is spherical if the subgroup $W_{T}$, generated by $T$, is finite. $\mathcal{S}$ denotes the set of spherical subsets of $S$, partially ordered by inclusion.

Let $A:=\mathbf{Z} W$ denote the group ring. Let $\left\{e_{w}\right\}_{w \in W}$ be its standard basis. For each $T \in \mathcal{S}$, put

$$
a_{T}:=\sum_{u \in W_{T}} e_{u} .
$$

$A^{T}$ denotes the right ideal $a_{T} A$. If $T \subset U \in \mathcal{S}$, then $a_{U} \in A^{T}$ (formula (3-2) of Section 3); hence, $A^{U} \subset A^{T}$. Let $A^{>T}$ be the right $W$-submodule spanned by the $A^{U}$, with $U \supsetneq T$.

For each $w \in W$, put $\operatorname{In}^{\prime}(w):=\{s \in S \mid l(s w)<l(w)\}$. It is a fact that $\operatorname{In}^{\prime}(w) \in \mathcal{S}$. Set $b_{w}^{\prime}:=a_{\operatorname{In}^{\prime}(w)} e_{w}$. We will show in Lemma 3.1 that $\left\{b_{w}^{\prime}\right\}_{w \in W}$ is also a basis for $A$. Define $\widehat{A}^{T}$ to be the $\mathbf{Z}$-submodule of $A$ spanned by $\left\{b_{w}^{\prime} \mid w \in W, \operatorname{In}^{\prime}(w)=T\right\}$. N.B. $\widehat{A}^{T}$ is not a $W$-submodule of $A$; however, $\widehat{A}^{T} \subset A^{T}$ and, as we shall see in Corollary 3.3, the natural map $\widehat{A}^{T} \rightarrow A^{T} / A^{>T}$ is an isomorphism of free abelian groups.

The Coxeter group $W$ acts properly and cocompactly as a group generated by reflections on a certain contractible complex $\Sigma$ (see Davis [3]). A fundamental domain for the $W$-action on $\Sigma$ is a finite simplicial complex $K$, defined as the geometric realization of the poset $\mathcal{S}$. Since $\emptyset$ is an initial element of $\mathcal{S}, K$ is contractible. For each $s \in S$, define $K_{s}$ to be the geometric realization of $\mathcal{S}_{\geq\{s\}}$, where $\mathcal{S}_{\geq\{s\}}:=\{T \in \mathcal{S} \mid s \in T\}$. $K_{S}$ is a subcomplex of $K$. For each $U \subset S$, put $K^{U}:=\bigcup_{s \in U} K_{s}$. The calculation of [5] was the following:

$$
H^{*}(W ; A)=H_{c}^{*}(\Sigma)=\bigoplus_{T \in \mathcal{S}} H^{*}\left(K, K^{S-T}\right) \otimes \widehat{A}^{T}
$$

We give a new proof of this calculation in Section 3 (Theorem 3.5). This proof involves showing that a certain coefficient system on $K$ decomposes as a direct sum and that cohomology groups with coefficients in the summands are precisely the terms on the right hand side of the above formula.

A similar formula should be true for buildings. By modifying the previous argument, we prove such a formula in Section 6 in the case of a locally finite, thick, right-angled building $\Phi$. In that section, $A$ stands for the abelian group of finitely supported 
$\mathbf{Z}$-valued functions on (the set of chambers of) $\Phi$. We define analogs of the $A^{T}$ and $\widehat{A}^{T}$ and in Theorem 6.6 we prove a formula of the form:

$$
H_{c}^{i}(|\Phi|) \cong \bigoplus_{T \in \mathcal{S}} H_{i}\left(K, K^{S-T}\right) \otimes \widehat{A}^{T}
$$

where $|\Phi|$ is the geometric realization of $\Phi$.

Let us return to the question of determining the $W$-action on cohomology. There is a decreasing filtration of right $W$-submodules of $A$ (where $A=\mathbf{Z} W$ ):

$$
F_{0} \supset \cdots F_{p} \supset \cdots
$$

Here $F_{p}$ is the submodule of $A$ spanned by the $A^{T}$ with $\operatorname{Card}(T) \geq p$. This induces a filtration of $H^{*}(W ; A)$. The main result of this paper is the following. (A more precise version of this result is stated as Theorem 4.5, below.)

Theorem In filtration degree $p$, the graded right $W$-module associated to the above filtration of $H^{*}(W ; A)$ is isomorphic to

$$
\bigoplus_{\substack{T \in \mathcal{S} \\ \operatorname{Card}(T)=p}} H^{*}\left(K, K^{S-T}\right) \otimes\left(A^{T} / A^{>T}\right)
$$

where $A^{T} / A^{>T}$ is the right $W$-module defined above.

Corollary $H^{*}(W ; A)$ is finitely generated as a $W$-module.

In view of Davis [5], Davis et al [6], Dymara and Januszkiewicz [8], Kazhdan and Lusztig [10] and Solomon [11], the above computation was a natural guess for the $W$-module structure on $H^{*}(W ; A)=H_{c}^{*}(\Sigma)$. In particular, in [6] we calculated the “weighted $L^{2}$-cohomology" of $\Sigma$ and obtained a very similar answer (provided the "weight" q lies in a certain range).

We actually proceed in somewhat more generality than indicated above. We consider an action of $W$ as a reflection group on a $\mathrm{CW}$ complex $\mathcal{U}$ with strict fundamental domain $X$ and then compute certain equivariant homology and cohomology groups of $\mathcal{U}$ with coefficients in $\mathbf{Z W}$. The equivariant (co)homology groups we are interested in have the following well known interpretations: $H_{*}^{W}(\mathcal{U} ; \mathbf{Z} W)=H_{*}(\mathcal{U})$ and when the action is proper and cocompact, $H_{W}^{*}(\mathcal{U} ; \mathbf{Z} W)=H_{c}^{*}(\mathcal{U})$. In Theorems 3.5 and 4.5 we prove formulas similar to the ones above for $H_{c}^{*}(\mathcal{U})$ and $H_{*}(\mathcal{U})$. In both cases the formulas involve terms of the form $H_{*}\left(X, X^{U}\right)$ or $H^{*}\left(X, X^{U}\right)$ with $U \subset S$. The difference is 
that in homology only the spherical subsets $U \in \mathcal{S}$ appear, while in cohomology only cospherical $U$ appear (ie, $S-U \in \mathcal{S}$ ). In the case of homology we recover the results of Davis [4].

When $\Phi$ is a building with a chamber transitive automorphism group $G$, one can try to calculate $H_{c}^{*}(|\Phi|)$ as a $G$-module. We make some comments about this in the last section. We first point out that the results of the previous sections hold when the group ring is replaced by the Hecke algebra $A_{\mathbf{q}}$ associated to $(W, S)$ and a multiparameter $\mathbf{q}$. When $\Phi$ is a building associated to a $B N$ pair and $\mathbf{q}$ its thickness, then $A_{\mathbf{q}}$ is closely related to the algebra of finitely supported functions on $\Phi$ and hence, to the cochains on $|\Phi|$. We state the natural conjecture (Conjecture 7.5) for the computation of $H_{c}^{*}(|\Phi|$ ) as a $G$-module and prove the cochain version of it as Theorem 7.3.

The first and the third authors were partially supported by NSF grant DMS 0405825. The second author was partially supported by KBN grant 2 PO3A 01725.

\section{Preliminaries}

Invariants and coinvariants Given a left $W$-module $M$ and a subset $T \subset S$, we have the $\mathbf{Z}$-submodule $M^{T} \subset M$ of the $W_{T}$-invariants defined by

$$
M^{T}:=M^{W_{T}}:=\left\{x \in M \mid w x=x \text { for all } w \in W_{T}\right\} .
$$

More generally, for any $\mathbf{Z}$-submodule $B \subset M$, put

$$
B^{T}:=B \cap M^{T} .
$$

For a right $W$-module $M$, the $W_{T}$-coinvariants are defined as a quotient $\mathbf{Z}$-module of $M$ :

$$
M_{T}:=M_{W_{T}}:=M \otimes_{W_{T}} \mathbf{Z} \cong M / M I_{T},
$$

where $I_{T}$ is the augmentation ideal of $\mathbf{Z} W_{T}$ and $\mathbf{Z}$ is the trivial $W_{T}$-module. For any $\mathbf{Z}$-submodule $B \subset M, B_{T}$ denotes the image of $B$ in $M_{T}$.

$\mathbf{Z}\left(W / W_{T}\right)$ denotes the (left) permutation module defined by the $W$-action on $W / W_{T}$.

Lemma 2.1 There are isomorphisms:

(i) $\operatorname{Hom}_{W}\left(\mathbf{Z}\left(W / W_{T}\right), M\right) \cong M^{T}$,

(ii) $M \otimes_{W} \mathbf{Z}\left(W / W_{T}\right) \cong M_{T}$, 
where $M$ is a left $W$-module in the first case and a right $W$-module in the second.

\section{Proof}

(i) $\operatorname{Hom}_{W}\left(\mathbf{Z}\left(W / W_{T}\right), M\right)$ can be identified with the set of $W$-equivariant functions $f: W / W_{T} \rightarrow M$. Because of equivariance, for any such $f, f\left(1 W_{T}\right) \in M^{T}$. Conversely, given any $x_{0} \in M^{T}$, the formula $f\left(w W_{T}\right)=w x_{0}$, gives a well-defined $f: W / W_{T} \rightarrow M$. So, $f \rightarrow f\left(1 W_{T}\right)$ defines an isomorphism from $\operatorname{Hom}_{W}\left(\mathbf{Z}\left(W / W_{T}\right), M\right)$ to $M^{T}$.

(ii) We have

$$
M \otimes_{W} \mathbf{Z}\left(W / W_{T}\right)=M \otimes_{W} \mathbf{Z} W \otimes_{W_{T}} \mathbf{Z}=M \otimes_{W_{T}} \mathbf{Z}=M_{T} .
$$

Remark 2.2 If $M$ is a bimodule, then the right $W$-action on $M$ gives both $\operatorname{Hom}_{W}\left(\mathbf{Z}\left(W / W_{T}\right), M\right)$ and $M^{T}$ the structure of right $W$-modules and the isomorphism in (i) is an isomorphism of right $W$-modules. Similarly, (ii) is an isomorphism of left $W$-modules.

The basic construction Suppose $X$ is a CW complex. Let $\mathcal{P}(X)$ denote the set of cells in $X$ and $X^{(i)}$ the set of $i$-cells. Given cells $c \in X^{(i)}$ and $c^{\prime} \in X^{(i-1)}$, let $\left[c: c^{\prime}\right]$ denote the incidence number. Write $c^{\prime}<c$ whenever the incidence number $\left[c: c^{\prime}\right]$ is nonzero. Extend this to a partial order on $\mathcal{P}(X)$.

A mirror structure on a CW complex $X$ is a family of subcomplexes $\left(X_{s}\right)_{s \in S}$ indexed by some set $S$ (which for us will always be the fundamental set of generators for the Coxeter group $W$ ). For each $T \subset S$, define subcomplexes of $X$ :

$$
X_{T}:=\bigcap_{s \in T} X_{s} \text { and } X^{T}:=\bigcup_{s \in T} X_{S}
$$

and set $X_{\emptyset}:=X$. For each cell $c$ in $X$, set

$$
S(c):=\left\{s \in S \mid c \subset X_{s}\right\} .
$$

Similarly, for each $x \in X, S(x):=\left\{s \in S \mid x \in X_{s}\right\}$.

From the above data we construct another $\mathrm{CW}$ complex $\mathcal{U}(W, X)$, with a cellular $W$-action, as follows. Give $W$ the discrete topology. Define an equivalence relation $\sim$ on $W \times X$ by $(w, x) \sim\left(w^{\prime}, x^{\prime}\right) \Longleftrightarrow x=x^{\prime}$ and $w W_{S(x)}=w^{\prime} W_{S\left(x^{\prime}\right)} . \mathcal{U}(W, X)$ is the quotient space $(W \times X) / \sim$. The $W$-action on it is the obvious one. $X$ is a fundamental domain for this action in the strict sense: the natural inclusion $X \hookrightarrow \mathcal{U}(W, X)$, which takes $x$ to the class of $(1, x)$, induces a homeomorphism $X \rightarrow \mathcal{U}(W, Z) / W$. 
When $X$ is the complex $K$, discussed in the Introduction, $\mathcal{U}(W, K)$ is the contractible complex $\Sigma$.

Coefficient systems A system of coefficients on a CW complex $X$ is a functor $\mathcal{F}$ from $\mathcal{P}(X)$ to the category of abelian groups. Here the poset $\mathcal{P}(X)$ is regarded as a category with $\operatorname{Hom}_{\mathcal{P}(X)}(c, d)$ equal to a singleton whenever $c \leq d$ and empty otherwise. The functor $\mathcal{F}$ will be contravariant whenever we are dealing with chains, homology or coinvariants and covariant in the case of cochains, cohomology or invariants. Define chains and cochains with coefficients in $\mathcal{F}$ by

$$
C_{i}(X ; \mathcal{F}):=\bigoplus_{c \in X^{(i)}} \mathcal{F}(c) \quad \text { and } \quad C^{i}(X ; \mathcal{F}):=\prod_{c \in X^{(i)}} \mathcal{F}(c) .
$$

We regard both $i$-chains and $i$-cochains as functions $f$ from $X^{(i)}$ to $\bigcup \mathcal{F}(c)$ such that $f(c) \in \mathcal{F}(c)$ for each $c \in X^{(i)}$. Boundary and coboundary maps are then defined by the usual formulas:

$$
\begin{aligned}
\partial(f)(c) & :=\sum[d: c] \mathcal{F}_{d c}(f(d)) \\
\delta(f)(c) & :=\sum[c: d] \mathcal{F}_{d c}(f(d))
\end{aligned}
$$

where, given an $i$-cell $c$, the first sum is over all $(i+1)$-cells $d$ which are incident to $c$ and the second sum is over all $(i-1)$-cells $d$ which are incident to $c$ and where $\mathcal{F}_{d c}: f(d) \rightarrow f(c)$ is the homomorphism corresponding to $d>c$ (in the first case) or $c>d$ (in the second).

Examples 2.3 Suppose $\left\{X_{s}\right\}_{s \in S}$ is a mirror structure on $X$.

(i) (Invariants) Given a left $W$-module $M$, define a (covariant) system of coefficients $\mathcal{I}(M)$ on $X$ by

$$
\mathcal{I}(M)(c):=M^{S(c)} .
$$

If $B \subset M$ is any $\mathbf{Z}$-submodule of $M$, then we have a sub-coefficient system $\mathcal{I}(B) \subset$ $\mathcal{I}(M)$, defined by $\mathcal{I}(B)(c):=B^{S(c)}$.

(ii) (Coinvariants) For a right $W$-module $M$, define a (contravariant) system of coefficients $\mathcal{C}(M)$ on $X$ by

$$
\mathcal{C}(M)(c):=M_{S(c)}
$$

Similarly, for any $\mathbf{Z}$-submodule $B$ of $M, \mathcal{C}(B)(c):=B_{S(c)}$. 
The following observation is the key to our results. Suppose $M$ is a left $W$-module and that we have a direct sum decomposition (of $\mathbf{Z}$-modules), $M=B \oplus C$, satisfying the following condition:

$$
M^{T}=B^{T} \oplus C^{T} \quad \text { for all } T \subset S .
$$

Then we have a direct sum decomposition of coefficient systems: $\mathcal{I}(M)=\mathcal{I}(B) \oplus \mathcal{I}(C)$. This leads to a decomposition of cochain groups: $C^{i}(X ; \mathcal{I}(M))=C^{i}(X ; \mathcal{I}(B)) \oplus$ $C^{i}(X ; \mathcal{I}(C)$ and a decomposition in cohomology:

$$
H^{*}(X ; \mathcal{I}(M))=H^{*}(X ; \mathcal{I}(B)) \oplus H^{*}(X ; \mathcal{I}(C))
$$

Similarly, suppose $M$ is a right $W$-module and $M=B \oplus C$ is a $\mathbf{Z}$-module decomposition satisfying:

$$
M_{T}=B_{T} \oplus C_{T} \quad \text { for all } T \subset S
$$

Then we get a decomposition of coefficient systems: $\mathcal{C}(M)=\mathcal{C}(B) \oplus \mathcal{C}(C)$ and a corresponding decomposition of homology groups:

$$
H_{*}(X ; \mathcal{C}(M))=H_{*}(X ; \mathcal{C}(B)) \oplus H_{*}(X ; \mathcal{C}(C)) .
$$

Equivariant (co)homology Given a discrete group $G$ acting cellularly on a CW complex $\Omega$, we will associate a certain equivariant homology and cohomology groups. Given a left $G$-module $M$, the $G$-equivariant cochains on $\Omega$ with coefficients in $M$ are defined by

$$
C_{G}^{i}(\Omega ; M):=\operatorname{Hom}_{G}\left(C_{i}(\Omega), M\right) .
$$

Similarly, if $M$ is a right $G$-module, we have the $G$-equivariant chains

$$
C_{i}^{G}(\Omega ; M):=M \otimes_{W} C_{i}(\Omega),
$$

where $C_{i}(\Omega)$ denotes the group of cellular $i$-chains on $\Omega$. (Some people think that "equivariant (co)homology" refers to the (co)homology of $\Omega \times{ }_{G} E G$ with local coefficients in $M$. However, there are other equivariant theories, for example, the one described above.)

If the $G$-action is free and the projection to the orbit space is a covering projection, then equivariant (co)chains on $\Omega$ are equal to the (co)chains on the orbit space with local coefficients in $M$. This is a useful viewpoint even when the action is not free. In general, $M$ does not induce a locally constant coefficient system on the orbit space. Rather, it induces a coefficient system on the orbit space thought of as an "orbihedron" or "complex of groups." The theory of such coefficient systems can be found in Haefliger 
[9]. These general coefficient systems on orbihedra are more general then the type considered above. (They correspond to "lax functors" rather than to functors.) However, as we shall see in Lemma 2.6, when $\Omega=\mathcal{U}(W, X)$, the induced coefficient system on $X$ coincides with one of the coefficient systems described in Examples 2.3.

In the case of coefficients in the group ring, we have the following well-known interpretation of equivariant (co)homology.

Proposition 2.4 Suppose $G$ acts cellularly on a $C W$ complex $\Omega$. Then

(i) $C_{*}^{G}(\Omega ; \mathbf{Z} G) \cong C_{*}(\Omega)$.

(ii) If the $G$-action is proper and there are only finitely many orbits of cells, then

$$
C_{G}^{*}(\Omega ; \mathbf{Z} G) \cong C_{c}^{*}(\Omega) .
$$

\section{Proof}

(i) $C_{i}^{G}(\Omega ; \mathbf{Z} G)=\mathbf{Z} G \otimes \mathbf{Z} G C_{i}(\Omega) \cong C_{i}(\Omega)$.

(ii) For any $G$-module $M, \operatorname{Hom}_{G}(M, \mathbf{Z} G) \cong \operatorname{Hom}_{c}(M, \mathbf{Z})$ (by Brown [2, Lemma 7.4, p age 208]) where $\operatorname{Hom}_{c}(M, \mathbf{Z})$ denotes the set of $\mathbf{Z}$-module maps $f: M \rightarrow \mathbf{Z}$ such that for each $m \in M, f(\mathrm{gm})=0$ for all but finitely many $g \in G$. Hence, $C_{G}^{i}(\Omega ; \mathbf{Z} G)=\operatorname{Hom}_{G}\left(C_{i}(\Omega), \mathbf{Z} G\right)=\operatorname{Hom}_{c}\left(C_{i}(\Omega), \mathbf{Z}\right)=C_{c}^{i}(\Omega)$.

Now let $\mathcal{U}=\mathcal{U}(W, X) . W$ acts properly on $\mathcal{U}$ with compact quotient if and only if $X$ is compact and $X_{U}=\emptyset$ whenever $U \notin \mathcal{S}$. In view of Proposition 2.4, when dealing with the cohomology of $\mathcal{U}$, we shall always assume that these conditions hold (ie, $X$ is compact and $X_{U}=\emptyset$ for all $U \notin \mathcal{S}$ ). However, in the formulas for the homology of $\mathcal{U}$, we need no extra assumptions on $X$. In the special case $\Omega=\mathcal{U}$, Proposition 2.4 becomes the following.

\section{Corollary 2.5}

(i) $C_{*}^{W}(\mathcal{U} ; \mathbf{Z} W) \cong C_{*}(\mathcal{U})$.

(ii) $C_{W}^{*}(\mathcal{U} ; \mathbf{Z} W) \cong C_{c}^{*}(\mathcal{U})$.

\section{Lemma 2.6}

(i) For any right $W$-module $M, C_{*}^{W}(\mathcal{U} ; M) \cong C_{*}(X ; \mathcal{C}(M))$.

(ii) For any left $W$-module $M, C_{W}^{*}(\mathcal{U} ; M) \cong C^{*}(X ; \mathcal{I}(M))$. 
Proof Any orbit of cells in $\mathcal{U}$ has the form $W c$ for some unique cell $c$ in $X$. As a $W$-set, this orbit is isomorphic to $W / W_{S(c)}$. Hence, using Lemma 2.1, we get

$$
\begin{aligned}
& C_{W}^{i}(\mathcal{U} ; M) \cong \bigoplus_{c \in X^{(i)}} M^{S(c)}=C^{i}(X ; \mathcal{I}(M)) \\
& C_{i}^{W}(\mathcal{U} ; M) \cong \bigoplus_{c \in X^{(i)}} M_{S(c)}=C_{i}(X ; \mathcal{C}(M)) .
\end{aligned}
$$

Remark The isomorphisms in Corollary 2.5 (ii) and Lemma 2.6 (ii) give

$$
C_{c}^{i}(\mathcal{U}) \cong C_{W}^{i}(\mathcal{U} ; \mathbf{Z} W) \cong C^{i}(X ; \mathcal{I}(\mathbf{Z} W)) \cong \bigoplus_{c \in X^{(i)}}(\mathbf{Z} W)^{S(c)}
$$

The composition of these gives an isomorphism $C_{c}^{i}(\mathcal{U}) \rightarrow \bigoplus_{c \in X^{(i)}}(\mathbf{Z W})^{S(c)}$, such that its component corresponding to $c \in X^{(i)}$ is given by

$$
f \rightarrow \sum_{w \in W} f\left(w^{-1} c\right) e_{w}
$$

where $\left(e_{w}\right)$ is the standard basis for $\mathbf{Z} W$. Similarly, the composition of the isomorphisms in Corollary 2.5 (i) and Lemma 2.6 (i) gives the obvious identification

$$
C_{i}(\mathcal{U}) \cong \bigoplus_{c \in X^{(i)}}(\mathbf{Z} W)_{S(c)}
$$

\section{Group ring coefficients}

Subsets of $W$ For any $U \subset S$, put

$$
\begin{aligned}
& \mathcal{X}_{U}:=\{w \in W \mid l(s w)>l(w) \text { for all } s \in U\} \\
& \mathcal{Y}_{U}:=\{w \in W \mid l(w s)>l(w) \text { for all } s \in U\}=\left(\mathcal{X}_{U}\right)^{-1}
\end{aligned}
$$

$\mathcal{X}_{U}$ (resp. $\left.\mathcal{Y}_{U}\right)$ is the set of elements in $W$ which are $(U, \emptyset)$-reduced (resp. $(\emptyset, U)$ reduced). $\mathcal{X}_{U}$ (resp. $\mathcal{Y}_{U}$ ) is a set of representatives for $W_{U} \backslash W$ (resp. $W / W_{U}$ ).

Given $w \in W$, set

$$
\begin{aligned}
\operatorname{In}(w) & :=\{s \in S \mid l(w s)<l(w)\}, \\
\operatorname{In}^{\prime}(w) & :=\{s \in S \mid l(s w)<l(w)\}=\operatorname{In}\left(w^{-1}\right) .
\end{aligned}
$$

$\operatorname{In}(w)$ (resp. $\left.\operatorname{In}^{\prime}(w)\right)$ is the set of letters of $S$ with which a reduced word for $w$ can end (resp. begin). By [3, Lemma 7.12], for any $w \in W, \operatorname{In}(w)$ is a spherical subset. We 
note that, for any $T \in \mathcal{S}$,

$$
\begin{aligned}
w_{T} \mathcal{X}_{T} & =\left\{w \in W \mid T \subset \operatorname{In}^{\prime}(w)\right\}, \\
\mathcal{Y}_{S-T} & =\{w \in W \mid T \supset \operatorname{In}(w)\},
\end{aligned}
$$

where $w_{T} \in W_{T}$ is the element of longest length. Thus, $w_{T} \mathcal{X}_{T}$ is also a set of representatives for $W_{T} \backslash W$.

Symmetrization and alternation From now on, except in Section $6, A$ denotes the group ring $\mathbf{Z} W$. For each spherical subset $T$ of $S$, define elements $a_{T}$ and $h_{T}$ in $A$ by

$$
a_{T}:=\sum_{w \in W_{T}} e_{w} \quad \text { and } \quad h_{T}:=\sum_{w \in W_{T}}(-1)^{l(w)} e_{w}
$$

called, respectively, symmetrization and alternation with respect to $T$. If $T \subset U \in \mathcal{S}$, define

$$
c_{(U, T)}:=\sum_{u \in \mathcal{X}_{T} \cap W_{U}} e_{u} \text { and } d_{(U, T)}:=\sum_{u \in \mathcal{Y}_{T} \cap W_{U}}(-1)^{l(w)} e_{u} .
$$

It is easily checked that

$$
a_{U}=a_{T} c_{(U, T)} \quad \text { and } \quad h_{U}=d_{(U, T)} h_{T} .
$$

For any subset $T$ of $S$, let $A^{T}$ denote the $W_{T}$-invariants in $A$, defined as in (2-1). Notice that $A^{T}$ is 0 if $T \notin \mathcal{S}$ and is equal to the right ideal $a_{T} A$ if $T \in \mathcal{S}$. Similarly, for $T \in \mathcal{S}$, define $H^{T}$ to be the left ideal $A h_{T}$ and to be 0 otherwise. By (3-2), $A^{U} \subset A^{T}$ and $H^{U} \subset H^{T}$ whenever $T \subset U$. Let $A_{T}$ denote the $W_{T}$-coinvariants, defined as in (2-2) and let $I_{T}$ denote the augmentation ideal of $\mathbf{Z} W_{T}$. For any $s \in S$, note that $A I_{\{s\}}=H^{\{s\}}$. Hence, $A_{\{s\}}=A / H^{\{s\}}$. More generally, for any $T \subset S$,

$$
A I_{T}=\sum_{s \in T} H^{\{s\}} \quad \text { so, } \quad A_{T}=A / \sum_{s \in T} H^{\{s\}} .
$$

Two bases for $A$ For each $w \in W$, define elements $b_{w}^{\prime}, b_{w} \in A$ by

$$
b_{w}^{\prime}:=a_{\operatorname{In}^{\prime}(w)} e_{w} \quad \text { and } \quad b_{w}:=e_{w} h_{\operatorname{In}(w)} .
$$

Lemma 3.1 $\left\{b_{w}^{\prime} \mid w \in W\right\}$ is a basis for $A$ (as a $\mathbf{Z}$-module). More generally, for any $T \in \mathcal{S},\left\{b_{w}^{\prime} \mid T \subset \operatorname{In}^{\prime}(w)\right\}$ is a basis for $A^{T}$.

Proof We first show $\left\{b_{w}^{\prime} \mid w \in W\right\}$ is a basis. The point is that the matrix which expresses the $b_{w}^{\prime}$ in terms of the $e_{w}$ has 1 's on the diagonal and is "upper triangular with respect to word length." In detail: first note that $b_{v}^{\prime}$ is the sum of $e_{v}$ with various 
$e_{w}$ having $l(w)<l(v)$. Suppose $\sum \beta_{w} b_{w}^{\prime}=0$ is a nontrivial linear relation. Let $v \in W$ be an element with $\beta_{v} \neq 0$ and $l(v)$ maximum. Since the coefficient of $e_{v}$ in the linear relation must be 0 , we have $\beta_{v}=0$, a contradiction. Similarly, one shows, by induction on word length, that each $e_{v}$ is a linear combination of $b_{w}^{\prime}$ with $l(w) \leq l(v)$. Hence, $\left\{b_{w}^{\prime}\right\}$ spans $A$.

To prove the second sentence, we must first show that $b_{w}^{\prime} \in A^{T}$ whenever $T \subset \operatorname{In}^{\prime}(w)$. If this condition holds, then, by (3-2),

$$
b_{w}^{\prime}=a_{\operatorname{In}^{\prime}(w)} e_{w}=a_{T} c_{\left(\operatorname{In}^{\prime}(w), T\right)} e_{w} \in A^{T} .
$$

Note that $T \subset \operatorname{In}^{\prime}(w)$ if and only if $w \in w_{T} \mathcal{X}_{T}$. Since, by the previous paragraph, $\left\{b_{w}^{\prime} \mid w \in w_{T} \mathcal{X}_{T}\right\}$ is linearly independent, it remains to show that it spans $A^{T}$. Since $w_{T} \mathcal{X}_{T}$ is a set of coset representatives for $W_{T} \backslash W$, a basis for $A^{T}$ is $\left\{a_{T} e_{w} \mid w \in w_{T} \mathcal{X}_{T}\right\}$. Let $\bar{e}_{w}:=c_{\left(\operatorname{In}^{\prime}(w), T\right)} e_{w}$. For $w \in w_{T} \mathcal{X}_{T}$, the matrix which expresses $\left\{\bar{e}_{w} \mid w \in w_{T} \mathcal{X}_{T}\right\}$ in terms of $\left\{e_{w} \mid w \in w_{T} \mathcal{X}_{T}\right\}$ has 1's on the diagonal and is upper triangular with respect to word length. So,

$$
\left\{a_{T} \bar{e}_{w} \mid w \in w_{T} \mathcal{X}_{T}\right\}=\left\{b_{w}^{\prime} \mid T \subset \operatorname{In}^{\prime}(w)\right\}
$$

is also a basis for $A^{T}$.

Lemma 3.2 $\left\{b_{w} \mid w \in W\right\}$ is a basis for $A$. More generally, for any subset $U$ of $S$, the projection $A \rightarrow A_{S-U}$ maps $\left\{b_{w} \mid U \supset \operatorname{In}(w)\right\}$ injectively to a basis for $A_{S-U}$.

Proof The proof of the first sentence is omitted since it is similar to that of the first sentence of the previous lemma.

Fix a subset $U \subset S$ and let $p: A \rightarrow A_{S-U}$ denote the projection. Since $A_{S-U}=$ $\mathbf{Z}\left(W / W_{S-U}\right),\left\{p\left(e_{w}\right) \mid w \in \mathcal{Y}_{S-U}\right\}$ is the obvious basis for $A_{S-U}$ (as a $\mathbf{Z}$-module). Any element $y \in A$ can be written in the form

$$
y=\sum_{w \in \mathcal{Y}_{S-U}} \sum_{u \in W_{S-U}} \alpha_{w u} e_{w u} .
$$

Moreover, $y \in A I_{S-U}=\operatorname{Ker}(p)$ if and only if $\sum_{u \in W_{S-U}} \alpha_{w u}=0$ for each $w \in \mathcal{Y}_{S-U}$. Let $y$ be an element in the submodule spanned by $\left\{b_{w} \mid U \supset \operatorname{In}(w)\right\}\left(=\left\{b_{w} \mid w \in\right.\right.$ $\left.\mathcal{Y}_{S-U}\right\}$ ), ie, let

$$
y=\sum_{w \in \mathcal{Y}_{S-U}} y_{w} b_{w}
$$

Suppose $p(y)=0$. Let $v \in \mathcal{Y}_{S-U}$ be such that $y_{v} \neq 0$ and $l(v)$ is maximum with respect to this property. Since $b_{v}$ is the sum of $e_{v}$ and \pm 1 times various $e_{w}$ with $l(w)<l(v)$, 
the coefficients $\alpha_{v u}$ in (3-3) are 0 for all $u \neq 1$ in $W_{S-U}$. So, $\sum \alpha_{v u}=0$ forces $\alpha_{v u}=0$, a contradiction. Thus, $\left\{p\left(b_{w}\right) \mid w \in \mathcal{Y}_{S-U}\right\}$ is linearly independent in $A_{S-U}$. The usual argument, using induction on word length, shows that $\left\{p\left(b_{w}\right) \mid w \in Y_{S-U}\right\}$ spans $A_{S-U}$.

In view of Lemmas 3.1 and 3.2, for each $T \in \mathcal{S}$, we define $\mathbf{Z}$-submodules of $A$ :

$$
\begin{gathered}
\widehat{A}^{T}:=\operatorname{Span}\left\{b_{w}^{\prime} \mid \operatorname{In}^{\prime}(w)=T\right\}, \\
\widehat{H}^{T}:=\operatorname{Span}\left\{b_{w} \mid \operatorname{In}(w)=T\right\} .
\end{gathered}
$$

A corollary to Lemma 3.1 is the following.

Corollary 3.3 For any $U \in \mathcal{S}$,

$$
A^{U}=\bigoplus_{T \in \mathcal{S}_{\geq U}} \widehat{A}^{T}
$$

Consequently, given $T \in \mathcal{S}$, for any $U \subset S$ we have:

$$
\left(\widehat{A}^{T}\right)^{U}= \begin{cases}\widehat{A}^{T}, & \text { if } U \subset T ; \\ 0, & \text { if } U \cap(S-T) \neq \emptyset .\end{cases}
$$

It follows that the direct sum decomposition in Corollary 3.3 satisfies (2-3) and hence, gives a decomposition of coefficient systems:

$$
\mathcal{I}(A)=\bigoplus_{T \in \mathcal{S}} \mathcal{I}\left(\widehat{A}^{T}\right)
$$

In terms of the $\widehat{H}^{T}$, the version of this we are interested in is the following:

$$
\left(\widehat{H}^{T}\right)_{U} \cong \begin{cases}\widehat{H}^{T}, & \text { if } U \subset S-T ; \\ 0, & \text { if } U \cap T \neq \emptyset .\end{cases}
$$

In the above formula, by writing $\left(\widehat{H}^{T}\right)_{U} \cong \widehat{H}^{T}$, we mean that the projection $A \rightarrow A_{U}$ maps $\widehat{H}^{T}$ isomorphically onto $\left(\widehat{H}^{T}\right)_{U}$. To see that $\left(\widehat{H}^{T}\right)_{U}=0$ when $U \cap T \neq \emptyset$, note that if $s \in T \cap U$, then $\widehat{H}^{T} \subset H^{s} \subset A I_{U}$.

The $\widehat{H}^{T}$ version of Corollary 3.3 is the following.

Corollary 3.4 For any $U \subset S$,

$$
A_{S-U}=\bigoplus_{T \in \mathcal{S}_{\leq U}}\left(\widehat{H}^{T}\right)_{S-U}
$$


So, the decomposition in Corollary 3.4 gives a decomposition of coefficient systems:

$$
\mathcal{C}(A)=\bigoplus_{T \in \mathcal{S}} \mathcal{C}\left(\widehat{H}^{T}\right)
$$

Hence, (2-4) and (2-5) apply to give the following calculation of (co)homology with group ring coefficients.

Theorem 3.5 Let $\mathcal{U}=\mathcal{U}(W, X)$. Then

$$
\begin{aligned}
& H_{c}^{i}(\mathcal{U}) \cong \bigoplus_{T \in \mathcal{S}} H^{i}\left(X, X^{S-T}\right) \otimes \widehat{A}^{T}, \\
& H_{i}(\mathcal{U}) \cong \bigoplus_{T \in \mathcal{S}} H_{i}\left(X, X^{T}\right) \otimes \widehat{H}^{T} .
\end{aligned}
$$

Proof To prove the first formula, note that by Proposition 2.4 and observation (2-4),

$$
C_{c}^{i}(\mathcal{U})=C^{i}(X ; \mathcal{I}(A))=\bigoplus_{T \in \mathcal{S}} C^{i}\left(X ; \mathcal{I}\left(\widehat{A}^{T}\right)\right)
$$

Given a cell $c \in X^{(i)}$, by (3-4),

$$
\left(\widehat{A}^{T}\right)^{S(c)}= \begin{cases}0, & \text { if } c \subset X^{S-T} \\ \widehat{A}^{T}, & \text { otherwise. }\end{cases}
$$

Hence,

$$
C^{i}\left(X ; \mathcal{I}\left(\widehat{A}^{T}\right)\right)=\left\{f: X^{(i)} \rightarrow \widehat{A}^{T} \mid f(c)=0 \text { if } c \subset X^{S-T}\right\}=C^{i}\left(X, X^{S-T}\right) \otimes \widehat{A}^{T} .
$$

Combining these formulas and taking cohomology, we get the first formula.

To prove the second formula, note that by Proposition 2.4 and observation (2-5),

$$
C_{i}(\mathcal{U})=C_{i}(X ; \mathcal{C}(A))=\bigoplus_{T \in \mathcal{S}} C_{i}\left(X ; \mathcal{C}\left(\widehat{H}^{T}\right)\right)
$$

Given a cell $c \in X^{(i)}$, by (3-6),

$$
\left(\widehat{H}^{T}\right)_{S(c)} \cong \begin{cases}0, & \text { if } c \subset X^{T} \\ \widehat{H}^{T}, & \text { otherwise. }\end{cases}
$$

Hence,

$$
C_{i}\left(X ; \mathcal{C}\left(\widehat{H}^{T}\right)\right)=\bigoplus_{\substack{c \in X^{(i)} \\ c \not \subset X^{T}}} \widehat{H}^{T} \cong C_{i}\left(X, X^{T}\right) \otimes \widehat{H}^{T} .
$$

Taking homology, we get the second formula.

Remark The first formula in Theorem 3.5 is one of the main results of [5]. (Actually, only a special case is stated in [5]; however, the general result is stated in [7].) The second formula is the main result of [4]. 


\section{The $W$-module structure of $H_{c}^{*}(\mathcal{U})$ and $H_{*}(\mathcal{U})$}

$A$ is a $W$-bimodule. So, $\mathcal{I}(A)$ is a system of right $W$-modules and $H^{*}(X ; \mathcal{I}(A))$ $\left(=H_{c}^{*}(\mathcal{U})\right)$ is a right $W$-module. Similarly, $\mathcal{C}(A)$ is a system of left $W$-modules and $H_{*}(X ; \mathcal{C}(A))\left(=H_{*}(\mathcal{U})\right)$ is a left $W$-module.

For each nonnegative integer $p$, define

$$
\begin{array}{rlrl}
F_{p} & :=\sum_{|T| \geq p} A^{T}, & E_{p} & :=\bigoplus_{|T|<p} \widehat{A}^{T}, \\
F_{p}^{\prime} & :=\sum_{|T| \geq p} H^{T}, & E_{p}^{\prime}:=\bigoplus_{|T|<p} \widehat{H}^{T},
\end{array}
$$

where $|T|:=\operatorname{Card}(T)$. As in Section 2, these give coefficient systems $\mathcal{I}\left(F_{p}\right)$ and $\mathcal{C}\left(F_{p}^{\prime}\right)$ on $X$. Note that $F_{p}$ is a right $W$-module and $\mathcal{I}\left(F_{p}\right)$ is a coefficient system of right $W$-submodules of $\mathcal{I}(A)$. Similarly, $\mathcal{C}\left(F_{p}^{\prime}\right)$ ) is a system of left $W$-modules. (However, $E_{p}$ and $E_{p}^{\prime}$ only have the structure of $\mathbf{Z}$ submodules of $A$.)

Lemma 4.1 We have decompositions (as $\mathbf{Z}$-modules):

(i) $A=F_{p} \oplus E_{p}$ and this induces a decomposition of coefficient systems, $\mathcal{I}(A)=$ $\mathcal{I}\left(F_{p}\right) \oplus \mathcal{I}\left(E_{p}\right)$.

(ii) $A=F_{p}^{\prime} \oplus E_{p}^{\prime}$ and this induces a decomposition of coefficient systems, $\mathcal{C}(A)=$ $\mathcal{C}\left(F_{p}^{\prime}\right) \oplus \mathcal{C}\left(E_{p}^{\prime}\right)$.

\section{Proof}

(i) By the second formula in Corollary 3.3, $F_{p}=\bigoplus_{|T| \geq p} \widehat{A}^{T}$; hence, by the first formula in the same corollary, $A=F_{p} \oplus E_{p}$. To get the decomposition of coefficient systems, we must show that $A^{U}=\left(F_{p}\right)^{U} \oplus\left(E_{p}\right)^{U}$ for all $U \subset S$. Since $A^{U}=\bigoplus_{T \supset U} \widehat{A}^{T}$,

$$
A^{U}=\bigoplus_{\substack{T \supset U \\|T| \geq p}} \widehat{A}^{T} \oplus \underset{\substack{T \supset U \\|T|<p}}{\bigoplus} \widehat{A}^{T} .
$$

Denote the first summation in (4-3) by $B$ and the second one by $C$.

Claim $B=\left(F_{p}\right)^{U}$. 
Proof of Claim Obviously, $B \subset\left(F_{p}\right)^{U}$. Let $x \in\left(F_{p}\right)^{U}$. Since $x \in F_{p}$, we have

$$
x=\sum_{|T| \geq p} \alpha^{T}
$$

where $\alpha^{T} \in \widehat{A}^{T}$. Since $x \in A^{U}$,

$$
x=\sum_{T \supset U} \beta^{T}
$$

where $\beta^{T} \in \widehat{A}^{T}$. But $A=\bigoplus_{T \subset S} \widehat{A}^{T}$, so the two decompositions of $x$ coincide. Therefore, $\alpha^{T}=0$ unless $T \supset U$ and

$$
x=\sum_{\substack{T \supset U \\|T| \geq p}} \alpha^{T} \in B,
$$

which proves that $\left(F_{p}\right)^{U} \subset B$.

Continuing with the proof of Lemma 4.1, note that a similar argument shows $\left(E_{p}\right)^{U}=C$. Hence, $A^{U}=\left(F_{p}\right)^{U} \oplus\left(E_{p}\right)^{U}$ and (i) is proved.

(ii) As before, by Corollary 3.4, $A=F_{p}^{\prime} \oplus E_{p}^{\prime}$. To get the decomposition of coefficient systems, we must show that $A_{S-U}=\left(F_{p}^{\prime}\right)_{S-U} \oplus\left(E_{p}^{\prime}\right)_{S-U}$ for all $U \subset S$. Since $A_{S-U}=\bigoplus_{T \subset U}\left(\widehat{H}^{T}\right)_{S-U}$,

$$
A_{S-U}=\bigoplus_{\substack{T \subset U \\|T| \geq p}}\left(\widehat{H}^{T}\right)_{S-U} \oplus \bigoplus_{\substack{T \subset U \\|T|<p}}\left(\widehat{H}^{T}\right)_{S-U}
$$

Denote the first summation in (4-4) by $B^{\prime}$ and the second one by $C^{\prime}$. We claim that $\left(F_{p}^{\prime}\right)_{S-U}=B^{\prime}$. Obviously, $B^{\prime} \subset\left(F_{p}^{\prime}\right)_{S-U}$. Any $x \in F_{p}$ can be written in the form

$$
x=\sum_{|T| \geq p} \gamma^{T},
$$

where $\gamma^{T} \in \widehat{H}^{T}$. Since $\gamma^{T} \in I_{S-U}$ whenever $T \cap(S-U) \neq \emptyset$, if $T \not \subset U$, we can set $\gamma^{T}=0$ without changing the congruence class of $x$ modulo $I_{S-U}$. So, putting

$$
y=\sum_{\substack{T \subset U \\|T| \geq p}} \gamma^{T}
$$

we have $y \equiv x \bmod I_{S-U}$ and $y \in B^{\prime}$. So, $\left(F_{p}^{\prime}\right)_{S-U} \subset B^{\prime}$. A similar argument shows $\left(E_{p}^{\prime}\right)_{S-U}=C^{\prime}$. Hence, $A_{S-U}=\left(F_{p}^{\prime}\right)_{S-U} \oplus\left(E_{p}^{\prime}\right)_{S-U}$ and (ii) is proved.

\section{Corollary 4.2}


(i) $\quad F_{p} \hookrightarrow A$ induces $H^{i}\left(X ; \mathcal{I}\left(F_{p}\right)\right) \hookrightarrow H^{i}(X ; \mathcal{I}(A))$ a $W$-equivariant embedding with image a $\mathbf{Z}$-module direct summand.

(ii) $\quad F_{p}^{\prime} \hookrightarrow A$ induces $H_{i}\left(X ; \mathcal{C}\left(F_{p}^{\prime}\right)\right) \hookrightarrow H_{i}(X ; \mathcal{C}(A))$ a $W$-equivariant embedding with image a $\mathbf{Z}$-module direct summand.

It follows that $F_{p+1} \hookrightarrow F_{p}$ induces $H^{*}\left(X ; \mathcal{I}\left(F_{p+1}\right)\right) \hookrightarrow H^{*}\left(X ; \mathcal{I}\left(F_{p}\right)\right)$, an embedding of right $W$-modules. This gives an associated graded group of right $W$-modules:

$$
H^{*}\left(X ; \mathcal{I}\left(F_{p}\right)\right) / H^{*}\left(X ; \mathcal{I}\left(F_{p+1}\right)\right) .
$$

Similarly, we have an embedding $H_{*}\left(X ; \mathcal{C}\left(F_{p+1}^{\prime}\right)\right) \hookrightarrow H_{*}\left(X ; \mathcal{C}\left(F_{p}^{\prime}\right)\right)$ of left $W$-modules and an associated graded group of left $W$-modules. Our goal in this section is to prove Theorem 4.5 below, which gives a complete computation of these graded $W$-modules.

For each $T \in \mathcal{S}$, put

$$
A^{>T}:=\sum_{U \supset T} A^{U} \quad \text { and } \quad H^{>T}:=\sum_{U \supset T} H^{U} .
$$

$A^{T} / A^{>T}$ is a right $W$-module and $H^{T} / H^{>T}$ is a left $W$-module.

Example 4.3 (The sign representation) $A^{\emptyset} / A^{>\emptyset}$ is isomorphic to $\mathbf{Z}$ as an abelian group. We can take the image $\bar{b}_{1}$ of the basis element $b_{1}\left(=e_{1}\right)$ as the generator. Since $a_{s} b_{1} \in A^{>\emptyset}, \bar{b}_{1} \cdot a_{s}=0$ for all $s \in S$. Hence, $\bar{b}_{1} \cdot s=-\bar{b}_{1}$. It follows that $W$ acts on $A^{\emptyset} / A^{>\emptyset}$ via the sign representation:

$$
\bar{b}_{1} \cdot w=(-1)^{l(w)} \bar{b}_{1} .
$$

Example 4.4 (The case of a finite Coxeter group) If $W$ is finite, then for any $T \subset S$, $A^{T} / A^{>T} \otimes \mathbf{Q}$ can be identified with a (right) $W$-submodule of the rational group algebra $\mathbf{Q} W$. Similarly, $H^{T} / H^{>T} \otimes \mathbf{Q}$ is a (left) $W$-submodule of $\mathbf{Q} W$. L Solomon proved in [11] that we have direct sum decompositions:

$$
\begin{aligned}
& \mathbf{Q} W=\bigoplus_{T \subset S} A^{T} / A^{>T} \otimes \mathbf{Q} \\
& \mathbf{Q} W=\bigoplus_{T \subset S} H^{T} / H^{>T} \otimes \mathbf{Q}
\end{aligned}
$$

Of course, there is no such decomposition over $\mathbf{Z}$. For an arbitrary Coxeter group $W$, a similar result for $L_{\mathbf{q}}^{2}(W)$ (the "q-weighted $L^{2}$-completion" of the regular representation) is proved in [6, Theorem 9.11]. 
Theorem 4.5 For each nonnegative integer $p$,

(i) there is an isomorphism of right $W$-modules:

$$
H^{*}\left(X ; \mathcal{I}\left(F_{p}\right)\right) / H^{*}\left(X ; \mathcal{I}\left(F_{p+1}\right)\right) \cong \bigoplus_{|T|=p} H^{*}\left(X, X^{S-T}\right) \otimes\left(A^{T} / A^{>T}\right)
$$

(ii) there is an isomorphism of left $W$-modules:

$$
H_{*}\left(X ; \mathcal{C}\left(F_{p}^{\prime}\right)\right) / H_{*}\left(X ; \mathcal{C}\left(F_{p+1}^{\prime}\right)\right) \cong \bigoplus_{|T|=p} H_{*}\left(X, X^{T}\right) \otimes\left(H^{T} / H^{>T}\right)
$$

In view of Corollary 2.5 and Lemma 2.6, this theorem gives a computation of the $W$-modules associated to the corresponding filtrations of $H_{c}^{*}(\mathcal{U})$ and $H_{*}(\mathcal{U})$. To prove the theorem we first need the following lemma.

Lemma 4.6 There are isomorphisms of $W$-modules:

$$
\psi: F_{p} / F_{p+1} \cong \bigoplus_{|T|=p} A^{T} / A^{>T} \quad \text { and } \quad \psi^{\prime}: F_{p}^{\prime} / F_{p+1}^{\prime} \stackrel{\cong}{\longrightarrow} \bigoplus_{|T|=p} H^{T} / H^{>T}
$$

Proof The inclusion $A^{T} \hookrightarrow F_{p}$ induces a map $A^{T} \rightarrow F_{p} / F_{p+1}$ and $A^{>T}$ is in the kernel; so, we get $A^{T} / A^{>T} \rightarrow F_{p} / F_{p+1}$. Therefore, we have a map of right $W$-modules:

$$
\phi: \bigoplus_{|T|=p} A^{T} / A^{>T} \rightarrow F_{p} / F_{p+1} .
$$

By Corollary 3.3, the inclusion $\widehat{A}^{T} \hookrightarrow A^{T}$ induces an isomorphism (of $\mathbf{Z}$-modules), $\widehat{A}^{T} \rightarrow A^{T} / A^{>T}$. Also, $F_{p}=\bigoplus_{|T|=p} \widehat{A}^{T} \oplus F_{p+1}$. So, we have a commutative diagram (of maps of $\mathbf{Z}$-modules):

$$
\begin{array}{ccc}
\bigoplus_{|T|=p} A^{T} / A^{>T} & \stackrel{\phi}{\longrightarrow} & F_{p} / F_{p+1} \\
& \bigoplus_{|T|=p} \widehat{A}^{T} &
\end{array}
$$

Since the two slanted arrows are bijections, so is $\phi$. Therefore, $\phi$ is an isomorphism of right $W$-modules. Put $\psi:=\phi^{-1}$.

The definition of the second isomorphism $\psi^{\prime}$ is similar. 
Here is some more notation. For any $T \subset S$, put

$$
Q_{\langle T\rangle}=A^{T} / A^{>T}, \quad Q_{\langle T\rangle}^{\prime}=H^{T} / H^{>T} .
$$

Since the right $W$-module $Q_{\langle T\rangle}$ is neither a left $W$-module or even a $\mathbf{Z}$-submodule of a left $W$-module, the definition of its (left) $W_{U}$-invariants as in (2-1) cannot be applied directly. Similarly, the definition of (right) coinvariants from (2-2) does not apply directly to $Q_{\langle T\rangle}^{\prime}$. Nevertheless, for each $U \subset S$, define:

$$
\begin{aligned}
& \left(Q_{\langle T\rangle}\right)^{U}:=\left(A^{T} \cap A^{U}\right) /\left(A^{>T} \cap A^{U}\right) \\
& \left(Q_{\langle T\rangle}^{\prime}\right)_{U}:=\left(H^{T}\right)_{U} /\left(H^{>T}\right)_{U}
\end{aligned}
$$

These give coefficient systems of $W$-modules on $X$ defined by

$$
\begin{aligned}
& \mathcal{I}\left(Q_{\langle T\rangle}\right)(c):=\left(Q_{\langle T\rangle}\right)^{S(c)} \\
& \mathcal{C}\left(Q_{\langle T\rangle}^{\prime}\right)(c):=\left(Q_{\langle T\rangle}^{\prime}\right)_{S(c)}
\end{aligned}
$$

respectively. As in (3-4) and (3-6),

$$
\begin{aligned}
& \left(Q_{\langle T\rangle}\right)^{U}= \begin{cases}A^{T} / A^{>T}, & \text { if } U \subset T ; \\
0, & \text { otherwise }\end{cases} \\
& \left(Q_{\langle T\rangle}^{\prime}\right)_{U}= \begin{cases}H^{T} / H^{>T}, & \text { if } U \subset S-T ; \\
0, & \text { otherwise. }\end{cases}
\end{aligned}
$$

\section{Lemma 4.7}

(i) $H^{i}\left(X ; \mathcal{I}\left(Q_{\langle T\rangle}\right)\right)=H^{i}\left(X, X^{S-T}\right) \otimes Q_{\langle T\rangle}$

(ii) $H_{i}\left(X ; \mathcal{C}\left(Q_{\langle T\rangle}^{\prime}\right)\right)=H^{i}\left(X, X^{T}\right) \otimes Q_{\langle T\rangle}^{\prime}$

\section{Proof}

(i) Using (4-5), we have

$$
\begin{aligned}
C^{i}\left(X ; \mathcal{I}\left(Q_{\langle T\rangle}\right)\right) & =\left\{f: X^{(i)} \rightarrow Q_{\langle T\rangle} \mid f(c)=0 \text { if } c \subset X^{S-T}\right\} \\
& =C^{i}\left(X, X^{S-T} ; Q_{\langle T\rangle}\right) \\
& \cong C^{i}\left(X, X^{S-T}\right) \otimes Q_{\langle T\rangle} .
\end{aligned}
$$


(ii) Using (4-6),

$$
\begin{aligned}
C_{i}\left(X ; \mathcal{C}\left(Q_{\langle T\rangle}^{\prime}\right)\right) & =\bigoplus_{c \in X^{(i)}}\left(Q_{\langle T\rangle}^{\prime}\right)_{S(c)} \\
& = \begin{cases}Q_{\langle T\rangle}^{\prime}, & \text { if } c \not \subset X^{T} ; \\
0, & \text { if } c \subset X^{T} ;\end{cases} \\
& =C_{i}\left(X, X^{T}\right) \otimes Q_{\langle T\rangle}^{\prime} .
\end{aligned}
$$

Lemma 4.8 For any $U \subset S$,

(i) the following sequence of right $W$-modules is exact,

$$
0 \longrightarrow\left(F_{p+1}\right)^{U} \longrightarrow\left(F_{p}\right)^{U} \stackrel{\tilde{\psi}}{\longrightarrow} \bigoplus_{|T|=p}\left(Q_{\langle T\rangle}\right)^{U} \longrightarrow 0,
$$

where $\tilde{\psi}$ is the map induced by $\psi$ and

(ii) the following sequence of left $W$-modules is exact,

$$
0 \longrightarrow\left(F_{p+1}^{\prime}\right)_{S-U} \longrightarrow\left(F_{p}^{\prime}\right)_{S-U} \stackrel{\tilde{\psi}^{\prime}}{\longrightarrow} \bigoplus_{|T|=p}\left(Q_{\langle T\rangle}^{\prime}\right)_{S-U} \longrightarrow 0
$$

where $\tilde{\psi}^{\prime}$ is the map induced by $\psi^{\prime}$.

Proof In the proof of Lemma 4.1, in formula (4-3), we showed

$$
\left(F_{p}\right)^{U}=\bigoplus_{\substack{|T| \geq p \\ T \supset U}} \widehat{A}^{T}
$$

Put

$$
B:=\bigoplus_{\substack{|T|=p \\ T \supset U}} \widehat{A}^{T}
$$

$B$ is a $\mathbf{Z}$-submodule of $\left(F_{p}\right)^{U}$ and it maps isomorphically onto $\left(F_{p}\right)^{U} /\left(F_{p+1}\right)^{U}$. The image of $B$ under $\psi$ is

$$
\bigoplus_{\substack{|T|=p \\ T \supset U}} A^{T} / A^{>T}=\bigoplus_{\substack{|T|=p \\ T \supset U}} Q_{\langle T\rangle}
$$

This proves (i).

The proof that the sequence in (ii) is short exact is similar. 


\section{Proof of Theorem 4.5}

(i) By Lemma 4.8 (i), we have a short exact sequence of coefficient systems on $X$ :

$$
0 \longrightarrow \mathcal{I}\left(F_{p+1}\right) \longrightarrow \mathcal{I}\left(F_{p}\right) \longrightarrow \bigoplus_{|T|=p} \mathcal{I}\left(Q_{\langle T\rangle}\right) \longrightarrow 0
$$

inducing a short exact sequence of cochain complexes:

$$
0 \longrightarrow C^{*}\left(X ; \mathcal{I}\left(F_{p+1}\right)\right) \longrightarrow C^{*}\left(X ; \mathcal{I}\left(F_{p}\right)\right) \longrightarrow \bigoplus_{|T|=p} C^{*}\left(X ; \mathcal{I}\left(Q_{\langle T\rangle}\right)\right) \longrightarrow 0 .
$$

By the argument for Corollary $4.2, H^{*}\left(X ; \mathcal{I}\left(F_{p+1}\right)\right) \rightarrow H^{*}\left(X ; \mathcal{I}\left(F_{p}\right)\right)$ is an injection onto a $(\mathbf{Z}$-module $)$ direct summand. Hence, the long exact sequence in cohomology decomposes into short exact sequences and we have:

$$
\begin{aligned}
H^{i}\left(X ; \mathcal{I}\left(F_{p}\right)\right) / H^{i}\left(X ; \mathcal{I}\left(F_{p+1}\right)\right) & \cong \bigoplus_{|T|=p} H^{i}\left(X ; \mathcal{I}\left(Q_{\langle T\rangle}\right)\right) \\
& \cong \bigoplus_{|T|=p} H^{i}\left(X, X^{S-T}\right) \otimes\left(A^{T} / A^{>T}\right)
\end{aligned}
$$

where the second isomorphism comes from Lemma 4.7 (i).

(ii) The proof of (ii) is similar.

Remark 4.9 The decreasing filtration $\supset F_{p} \supset F_{p+1} \cdots$ of (4-1) gives a decreasing filtration of cochain complexes

$$
\cdots \supset C^{*}\left(X ; \mathcal{I}\left(F_{p}\right)\right) \supset C^{*}\left(X ; \mathcal{I}\left(F_{p+1}\right)\right) \cdots
$$

So, the quotient cochain complexes have the form $C^{*}\left(X ; \mathcal{I}\left(F_{p}\right) / \mathcal{I}\left(F_{p+1}\right)\right)$. Taking homology, we get a spectral sequence with $E_{1}$-term:

$$
E_{1}^{p q}:=H^{p+q}\left(X ; \mathcal{I}\left(F_{p}\right) / \mathcal{I}\left(F_{p+1}\right)\right)
$$

It converges to:

$$
E_{\infty}^{p q}:=\frac{H^{p+q}\left(X ; \mathcal{I}\left(F_{p}\right)\right)}{\operatorname{Im}\left(H^{p+q}\left(X ; \mathcal{I}\left(F_{p+1}\right)\right)\right)}
$$

So, the import of Theorem 4.5 is that $E_{1}^{p q}=E_{\infty}^{p q}$. 


\section{$5 \quad H^{*}(W ; \mathbf{Z} W)$}

Let $K$ denote the geometric realization of the poset $\mathcal{S}$ of spherical subsets. (The simplicial complex $K$ is contractible because it is a cone; the cone point corresponds to the minimum element $\emptyset \in \mathcal{S}$.) For each $s \in S$, define a subcomplex $K_{s} \subset K$ as the geometric realization of $\mathcal{S}_{\geq\{s\}}$. Put $\Sigma:=\mathcal{U}(W, K)$. (Alternatively, $\Sigma$ could be described as the geometric realization of the poset $W \mathcal{S}$ of all "spherical cosets," ie, the poset of all cosets of the form $w W_{T}$, with $w \in W$ and $T \in \mathcal{S}$.)

By construction $W$ acts properly on $\Sigma$. It is proved in [3] that $\Sigma$ is contractible. Hence,

$$
H^{*}(W ; \mathbf{Z} W)=H_{c}^{*}(\Sigma) .
$$

As before, $A:=\mathbf{Z} W$. The filtration $A=F_{0} \supset \cdots F_{p} \supset \cdots$ gives $H_{c}^{*}(\Sigma)=H^{*}(K ; \mathcal{I}(A))$ the structure of a graded $W$-module. As in (4-7), let $E_{\infty}^{p q}$ is the right $W$-module in filtration degree $p$ associated to $H^{p+q}(K ; \mathcal{I}(A))$. Theorem 4.5 then has the following corollary.

Theorem 5.1 The associated graded group of $H^{p+q}(W ; \mathbf{Z W})$ is given, as a right $W$-module, by

$$
E_{\infty}^{p q}=\bigoplus_{|T|=p} H^{p+q}\left(K, K^{S-T}\right) \otimes\left(A^{T} / A^{>T}\right)
$$

It follows from Theorem 3.5 that we have a direct sum decomposition of $\mathbf{Z}$-modules:

$$
H_{c}^{*}(\Sigma) \cong \bigoplus_{T \in \mathcal{S}} H^{*}\left(K, K^{S-T}\right) \otimes \widehat{A}^{T} .
$$

In view of Theorem 5.1, it is natural to conjecture that $H_{c}^{*}(\Sigma)$ decomposes as above into a direct sum of right $W$-modules. However, in general, there is no such decomposition, as we can see by considering the following example.

Example 5.2 Suppose $W$ is the free product of 3 copies of $\mathbf{Z} / 2$. Then $K$ is the cone on 3 points. So, it has 3 edges. By Theorem $4.5, H^{1}\left(K, K^{S}\right) \otimes A / A^{>\emptyset}$ is a quotient of $H_{c}^{1}(\Sigma)$. Let $x \in C^{1}(K)$ be a cochain (= cocycle) which evaluates to 1 on one of the edges, call it $c$, and to 0 on the other two edges. Choose $s \in S$ which is not a vertex of $c$. Let $y$ denote the image of $x \otimes 1$ in $H^{1}\left(K, K^{S}\right) \otimes A / A^{>\emptyset}$. By Example 4.3, $A / A^{>\emptyset}$ has rank 1 as an abelian group and the $W$-action on it is given by the sign representation. Hence, $y \cdot s=-y$ in $H^{1}\left(K, K^{S}\right) \otimes A / A^{>\emptyset}$. Suppose we had a $W$-equivariant splitting $\varphi: H^{1}\left(K, K^{S}\right) \otimes A / A^{>\emptyset} \rightarrow H_{c}^{1}(\Sigma)$. When regarded as an 


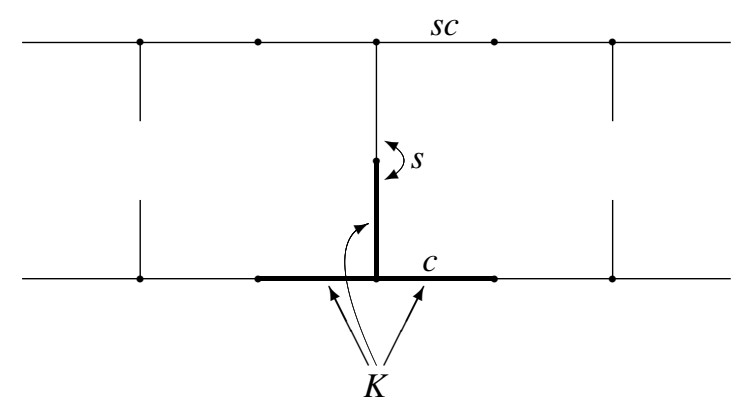

Figure 1: Cocycles $x$ and $x \cdot s$

element of $C_{c}^{1}(\Sigma), x+x \cdot s$ represents $\varphi(y+y \cdot s)$ in $H_{c}^{1}(\Sigma)$, ie, it represents 0 . But $x$ and $-x \cdot s$ are not cohomologous cocycles in $C_{c}^{1}(\Sigma)$. (One can see this by noting that there is a line ( $=$ infinite 1 -cycle) on which $x$ evaluates to 1 and $x \cdot s$ to 0 ; see Figure 1.) Hence, there can be no such splitting $\varphi$.

\section{Buildings}

A chamber system over $S$ is a set $\Phi$ of chambers together with a family of equivalence relations on $\Phi$ indexed by $S$. Two chambers are s-equivalent if they are equivalent via the equivalence relation with index $s$; they are $s$-adjacent if they are $s$-equivalent and not equal. A gallery in $\Phi$ is a finite sequence of chambers $\left(\varphi_{0}, \ldots, \varphi_{k}\right)$ such that $\varphi_{j-1}$ is adjacent to $\varphi_{j}, 1 \leq j \leq k$. The type of this gallery is the word $\mathbf{s}=\left(s_{1}, \ldots, s_{k}\right)$ where $\varphi_{j-1}$ is $s_{j}$-adjacent to $\varphi_{j}$. If each $s_{j}$ belongs to a given subset $T$ of $S$, then the gallery is a $T$-gallery. A chamber system is connected (resp. T-connected) if any two chambers can be joined by a gallery (resp. a $T$-gallery). The $T$-connected components of a chamber system $\Phi$ are its residues of type $T$. Given $\varphi \in \Phi, \operatorname{Res}(\varphi, T)$ denotes the residue of type $T$ containing $\varphi$.

Suppose $(W, S)$ is a Coxeter system and $M=\left(m_{s t}\right)$ its Coxeter matrix. A building of type $(W, S)$ (or of type $M$ ) is a chamber system $\Phi$ over $S$ such that

(i) for all $s \in S$, each $s$-equivalence class contains at least two chambers, and

(ii) there exists a $W$-valued distance function $\delta: \Phi \times \Phi \rightarrow W$. (This means that given a reduced word $\mathbf{s}$ for an element $w \in W$, chambers $\varphi$ and $\varphi^{\prime}$ can be joined by a gallery of type $\mathbf{s}$ if and only if $\delta\left(\varphi, \varphi^{\prime}\right)=w$. 
A residue of type $T$ in a building is itself a building; its type is $\left(W_{T}, T\right)$. A building of type $(W, S)$ is spherical if $W$ is finite. A building has finite thickness if all $s$-equivalence classes are finite, for all $s \in S$. (This implies all spherical residues are finite.) A building is thick if all $s$-equivalence classes have at least 3 elements, for all $s \in S$.

Fix a base chamber $\varphi_{0} \in \Phi$. The folding map, $\pi: \Phi \rightarrow W$, centered at $\varphi_{0}$ is defined by $\pi(\varphi):=\delta\left(\varphi_{0}, \varphi\right)$.

A Coxeter matrix $M$ is right-angled if all its off-diagonal entries are 2 or $\infty$. A building is right-angled if its Coxeter matrix is.

Example 6.1 (Right-angled spherical buildings) If $(W, S)$ is right-angled and spherical, then it has the form $W=W_{s_{1}} \times \cdots \times W_{s_{n}}$, with $S=\left\{s_{1}, \ldots s_{n}\right\}$ (ie, $W \cong(\mathbf{Z} / 2)^{S}$ ). A building of type $\left(W_{s_{i}},\left\{s_{i}\right\}\right)$ is simply a set $\Phi_{i}$ with at least 2 elements (it is thick if it has at least 3 elements). It follows that any right-angled spherical building $\Phi$ has the form $\Phi=\Phi_{1} \times \cdots \times \Phi_{n}$. Fix a base chamber $\varphi_{0}=\left(x_{1}, \ldots, x_{n}\right) \in \Phi$, giving us a folding map $\pi: \Phi \rightarrow W$. Let $L_{\Phi}$ be the set of $\varphi \in \Phi$ such that $\pi(\varphi)$ is the longest element in $W$. Clearly, $L_{\Phi}=\left(\Phi_{1}-x_{1}\right) \times \cdots \times\left(\Phi_{n}-x_{n}\right)$. So, when $\Phi$ is thick, $L_{\Phi}$ is also a right-angled spherical building.

In what follows $\Phi$ is a building of finite thickness and type $(W, S) . \pi: \Phi \rightarrow W$ is a folding map. For the remainder of this section, $A$ denotes the abelian group of finitely supported ( $\mathbf{Z}$-valued) functions on $\Phi$ and for any subset $T$ of $S, A^{T} \subset A$ denotes the subgroup of functions which are constant on $T$-residues. For each $w \in W$, put $\operatorname{Out}(w):=S-\operatorname{In}(w)$, where $\operatorname{In}(w)$ was defined in Section 3.

Lemma 6.2 Suppose that $(\Phi, \pi)$ is a spherical building equipped with a folding map. For $\varphi \in \Phi$, let $g_{\varphi}$ be the characteristic function of $\operatorname{Res}(\varphi, \operatorname{Out}(\pi(\varphi)))$. For any $T \subset S$, put $B^{T}=\left\{g_{\varphi} \mid \operatorname{Out}(\pi(\varphi)) \supset T\right\}$. Then $B^{T}$ is a basis of $A^{T}$.

Proof First we check that $\left\{g_{\varphi}\right\}_{\varphi \in \Phi}$ is linearly independent. Suppose we have a linear relation $\sum_{\varphi \in \Phi} \alpha_{\varphi} g_{\varphi}=0$. Choose $\psi$ with shortest $\pi(\psi)$ such that $\alpha_{\psi} \neq 0$. Then $0=\left(\sum_{\varphi \in \Phi} \alpha_{\varphi} g_{\varphi}\right)(\psi)=\alpha_{\psi}$, contradiction.

Put $C^{T}:=\{\varphi \in \Phi \mid \varphi$ is the shortest element of $\operatorname{Res}(\varphi, T)\}$. Then $C^{T}$ is a set of representatives for the set of $T$-residues of $\Phi$. Notice that $T \subset \operatorname{Out}(\pi(\varphi))$ if and only if $\varphi \in C^{T}$. Therefore, $\operatorname{Card}\left(B^{T}\right)=\operatorname{Card}\left(C^{T}\right)=\operatorname{rank}\left(A^{T}\right)$. Let $e_{R}$ denote the characteristic function of a residue $R$ in $\Phi$. Put $R_{\varphi}:=\operatorname{Res}(\varphi, T)$. The standard basis for $A^{T}$ is $\left\{e_{R_{\varphi}}\right\}_{\varphi \in C^{T}}$. Suppose $T \subset \operatorname{Out}(\pi(\varphi))$. Then $g_{\varphi}=\sum e_{R}$ where $R$ ranges 
over the $T$-residues in $\operatorname{Res}(\varphi, \operatorname{Out}(\pi(\varphi)))$. In other words, the matrix which expresses $\left\{g_{\varphi}\right\}_{\varphi \in C^{T}}$ in terms of the $e_{R_{\varphi}}$ has 1 's on the diagonal and is upper triangular when $C^{T}$ is ordered with respect to $l(\pi(\varphi))$. Hence, $B^{T}$ is also a basis for $A^{T}$

Now suppose $\Phi$ is right-angled, thick and infinite. Let $R$ be a spherical residue in $\Phi$, and $U \subset S$ its type. Let $L_{R}$ denote the set of chambers $\varphi \in R$ which have the longest possible $\pi(\varphi)$. By Example 6.1, $L_{R}$ is a right-angled spherical building of type $\left(W_{U}, U\right)$. For each spherical residue $R$, fix a folding map $\pi_{L_{R}}: L_{R} \rightarrow W_{U}$. Note that $\pi_{L_{R}}$ is totally different from (the restriction of) $\pi$.

Definition 6.3 Let $\varphi \in \Phi, U=\operatorname{In}(\pi(\varphi))$ and $R=R_{\varphi}:=\operatorname{Res}(\varphi, U)$. Then $\varphi \in L_{R}$. Define $f_{\varphi}$ to be the characteristic function of $\operatorname{Res}_{\Phi}\left(\varphi, \operatorname{Out}\left(\pi_{L_{R}}(\varphi)\right)\right)$, where $\operatorname{Out}()$ is computed in $W_{U}$.

We claim that the restriction of $f_{\varphi}$ to $L_{R}$ is the function $g_{\varphi}$ obtained by applying Lemma 6.2 to $\left(L_{R}, \pi_{L_{R}}\right)$. To see this, it is enough to check the following fact: for any $T \subset U$, the intersection of a $T$-residue in $\Phi$ with $L_{R}$ is either empty or is a $T$-residue in $L_{R}$. Let $\psi \in L_{R}$ and let $p: R \rightarrow W_{U}$ be the $\psi$-based folding map. Then $p$ maps $\operatorname{Res}_{R}(\psi, T)$ and $\operatorname{Res}_{L_{R}}(\psi, T)$ onto $W_{T}$, while any other $T$-residue in $L_{R}$ is mapped onto a nontrivial coset of $W_{T}$ in $W_{U}$. It follows that $\operatorname{Res}_{R}(\psi, T) \cap L_{R}=\operatorname{Res}_{L_{R}}(\psi, T)$.

Observe that if $g_{\varphi}$ is constant on $T$-residues in $L_{R}$, then $T \subset \operatorname{Out}\left(\pi_{L_{R}}(\varphi)\right)$ and hence, $f_{\varphi}$ is constant on $T$-residues in $\Phi$. Also, the support of $f_{\varphi}$ is contained in $R_{\varphi}$.

Not every spherical residue is of the form $R_{\varphi}$. Call a spherical residue $R$ greedy, if for some (hence, every) $\varphi \in L_{R}$, we have $R=\operatorname{Res}(\varphi, \operatorname{In}(\pi(\varphi))$ ). For a greedy residue $R$ of type $U$ and a subset $T \subset U$, put $B^{R, T}=\left\{f_{\varphi} \mid \varphi \in L_{R}\right.$, Out $\left.\left(\pi_{L_{R}}(\varphi)\right) \supset T\right\}$ (for convenience, put $B^{R, T}=\emptyset$ if $\left.T \not \subset U\right)$. Then $\left\{\left.f\right|_{L_{R}} \mid f \in B^{R, T}\right\}$ coincides with the basis $B^{T}$ associated to $\left(L_{R}, \pi_{L_{R}}\right)$ in Lemma 6.2. Therefore, $B^{R, T}$ is linearly independent. In particular, the set $B^{R, \emptyset}=\left\{f_{\varphi} \mid \varphi \in L_{R}\right\}$ is linearly independent.

For any spherical $T \subset S$ we put $B^{T}=\bigcup B^{R, T}$, where the union is over all greedy residues. Also put $B=B^{\emptyset}=\left\{f_{\varphi} \mid \varphi \in \Phi\right\}$. The next result is the analog of Lemma 3.1.

Theorem 6.4 For each $T \in \mathcal{S}, B^{T}$ is a basis of $A^{T}$.

Proof First we check that $B$ is linearly independent. Let $\sum_{\varphi \in \Phi} \alpha_{\varphi} f_{\varphi}=0$. Suppose $\psi \in \Phi$ is an element with $\alpha_{\psi} \neq 0$ and maximum $l(\pi(\psi))$. Let $R=\operatorname{Res}(\psi, \operatorname{In}(\pi(\psi)))$. 
Then $\left.\sum_{\varphi \in \Phi} \alpha_{\varphi} f_{\varphi}\right|_{L_{R}}=\left.\sum_{\varphi \in L_{R}} \alpha_{\varphi} f_{\varphi}\right|_{L_{R}}$. Since the set $\left\{f_{\varphi} \mid \varphi \in L_{R}\right\}$ is a linearly independent, we have $\alpha_{\varphi}=0$ for $\varphi \in L_{R}$. In particular $\alpha_{\psi}=0$, contradiction.

Suppose now that $f \in A^{T}$ is not a linear combination of elements of $B^{T}$. Choose such an $f$ with minimum $M:=\max \{l(\pi(\varphi)) \mid f(\varphi) \neq 0\}$. Let $\psi \in \Phi$ be such that $f(\psi) \neq 0$ and $\ell(\pi(\psi))=M$. Let $R=\operatorname{Res}(\psi, \operatorname{In}(\pi(\psi)))$. Clearly, $U=\operatorname{In}(\pi(\psi)) \supset T$. Let $\left(R_{i}\right)_{i=0, \ldots, N}$ be the collection of $U$-residues in $\Phi$ that are $U$-connected components of $\pi^{-1}\left(\pi(R)\right.$ ) (where $R_{0}=R$ ). These residues are pairwise disjoint. We also note that they are greedy. Indeed, (1) $R_{0}$ is greedy by its definition, (2) the notion of greediness for a residue depends only on its image under $\pi$ and (3) $\pi\left(R_{i}\right)=\pi\left(R_{0}\right)$. The restrictions to $L_{R_{i}}$ of functions from $B^{R_{i}, T}$ form a basis of the space of functions on $L_{R_{i}}$ constant on $T$-residues - in fact, this is exactly the basis produced by applying Lemma 6.2 to $L_{R_{i}}$. Since the restriction of $f$ to $L_{R_{i}}$ is constant on $T$-residues, there exists $f_{R_{i}} \in \operatorname{Span}\left(B^{R_{i}, T}\right)$ whose restriction to $L_{R_{i}}$ coincides with that of $f$. Let $\widetilde{f}=f-\sum_{i=0}^{N} f_{R_{i}}$. Then $\widetilde{f}$ is in $A^{T}$, is not in $\operatorname{Span}\left(B^{T}\right)$ and has value 0 on $\pi^{-1}(\pi(\psi))$. Observe also that if $\varphi \notin \pi^{-1}(\pi(\psi))$ and $\ell(\pi(\varphi))=M$, then $\widetilde{f}(\varphi)=f(\varphi)$. Therefore, we can repeat our procedure for all such $\varphi$, and finally obtain a counterexample to $A^{T}=\operatorname{Span}\left(B^{T}\right)$ with smaller $M$, a contradiction.

Put $\widehat{B}^{T}:=B^{T}-\bigcup_{U \supset T} B^{U}$ and $\widehat{A}^{T}:=\operatorname{Span}\left(\widehat{B}^{T}\right)$. Just as in Section 3, Theorem 6.4 has the following corollary.

Corollary 6.5 (Compare Corollary 3.3)

$$
A^{U}=\bigoplus_{T \supset U} \widehat{A}^{T} .
$$

As in Examples 2.3, given a mirror structure $\left(X_{S}\right)_{s \in S}$ on a CW complex $X$, we get a covariant coefficient system $\mathcal{I}(A)$ on $X$ defined by $\mathcal{I}(A)(c):=A^{S(c)}$. As in (3-5), Corollary 6.5 means that we have a decomposition of coefficient systems:

$$
\mathcal{I}(A)=\bigoplus_{T \in \mathcal{S}} \mathcal{I}\left(\widehat{A}^{T}\right)
$$

where $\mathcal{I}\left(\widehat{A}^{T}\right)(c):=\widehat{A}^{T} \cap A^{S(c)}$.

The geometric realization of a building Given a CW complex $X$ with mirror structure, define an equivalence relation $\sim$ on $\Phi \times X$ by $(\varphi, x) \sim\left(\varphi^{\prime}, x^{\prime}\right)$ if and only if $x=x^{\prime}$ and $\delta\left(\varphi, \varphi^{\prime}\right) \in W_{S(x)}$. The $X$-realization of $\Phi$, denoted $\mathcal{U}(\Phi, X)$, is defined by

$$
\mathcal{U}(\Phi, X):=(\Phi \times X) / \sim .
$$


( $\Phi$ has the discrete topology.) When $X=K$ (the geometric realization of $\mathcal{S}$ ), $\mathcal{U}(\Phi, K)$ is denoted by $|\Phi|$ and called the geometric realization of $\Phi$.

As in Corollary 2.5 and Lemma 2.6, identify $C^{*}(\mathcal{U}(\Phi, X))$ with $C^{*}(X ; \mathcal{I}(A))$. Using $(6-1)$, the proof of Theorem 3.5 goes through to give the following.

Theorem 6.6 Suppose $\Phi$ is a right-angled, thick, infinite building. Then

$$
H_{c}^{i}(\mathcal{U}(\Phi, X)) \cong \bigoplus_{T \in \mathcal{S}} H^{i}\left(X, X^{S-T}\right) \otimes \widehat{A}^{T}
$$

In particular,

$$
H_{c}^{i}(|\Phi|) \cong \bigoplus_{T \in \mathcal{S}} H_{i}\left(K, K^{S-T}\right) \otimes \widehat{A}^{T} .
$$

Remark A similar result for any building of finite thickness is claimed in [7, Theorem 5.8]; however, there is a mistake in the proof.

\section{Hecke algebra coefficients}

In this section we work over the rational numbers $\mathbf{Q}$ rather than $\mathbf{Z}$.

Let $i: S \rightarrow I$ be a function to some index set $I$ such that $i(s)=i\left(s^{\prime}\right)$ whenever $s$ and $s^{\prime}$ are conjugate in $W$. Let $\mathbf{q}=\left(q_{i}\right)_{i \in I}$ be a fixed $I$-tuple of rational numbers. Write $q_{s}$ instead of $q_{i(s)}$. If $s_{1} \cdots s_{l}$ is a reduced expression for an element $w \in W$, then the number $q_{s_{1}} \cdots q_{s_{l}}$ is independent of the choice of reduced expression. We write it as $q_{w}$. The Hecke algebra $A_{\mathbf{q}}$ of $W$ is a deformation of the group algebra $\mathbf{Q} W$ which is equal to $\mathbf{Q} W$ when each $q_{s}=1$. As a rational vector space, it has the same basis $\left\{e_{w}\right\}_{w \in W}$ as does $\mathbf{Q} W$. Multiplication is determined by the rules:

$$
\begin{aligned}
e_{w} e_{w^{\prime}} & =e_{w w^{\prime}}, \quad \text { if } l\left(w w^{\prime}\right)=l(w)+l\left(w^{\prime}\right) \\
e_{s}^{2} & =\left(q_{s}-1\right) e_{s}+q_{s} .
\end{aligned}
$$

Given a special subgroup $W_{T}, A_{\mathbf{q}}\left(W_{T}\right)$ denotes the Hecke algebra of $W_{T}$. It is a subalgebra of $A_{\mathbf{q}}$. There are ring homomorphisms $\alpha: A_{\mathbf{q}}\left(W_{T}\right) \rightarrow \mathbf{Q}$ and $\beta: A_{\mathbf{q}}\left(W_{T}\right) \rightarrow$ $\mathbf{Q}$, defined by $\alpha\left(e_{w}\right):=q_{w}$ and $\beta\left(e_{w}\right):=(-1)^{l(w)}$, respectively. Given a left $A_{\mathbf{q}}$-module $M$ and a subset $T$ of $S$, put

$$
M^{T}:=\left\{x \in M \mid a x=\alpha(a) x \text { for all } a \in A_{\mathbf{q}}\left(W_{T}\right)\right\} .
$$

This gives a coefficient system $\mathcal{I}(M)$ on $X$ in the same way as Examples 2.3. 
As in [6], for each $T \in \mathcal{S}$, we modify the formulas in (3-1) to define elements $a_{T}$ and $h_{T}$ in $A_{\mathbf{q}}$ by

$$
a_{T}:=\frac{1}{W_{T}(\mathbf{q})} \sum_{w \in W_{T}} e_{w} \quad \text { and } \quad h_{T}:=\frac{1}{W_{T}\left(\mathbf{q}^{-1}\right)} \sum_{w \in W_{T}}(-1)^{l(w)} q_{w}^{-1} e_{w}
$$

where

$$
W_{T}(\mathbf{q}):=\sum_{w \in W_{T}} q_{w}, \quad \text { and } \quad W_{T}\left(\mathbf{q}^{-1}\right):=\sum_{w \in W_{T}} q_{w}^{-1} .
$$

Put $A_{\mathbf{q}}^{T}:=a_{T} A_{\mathbf{q}}, H_{\mathbf{q}}^{T}:=A_{\mathbf{q}} h_{T}$. (If $T \notin \mathcal{S}, A_{\mathbf{q}}^{T}:=0, H_{\mathbf{q}}^{T}:=0$.)

For each subset $U$ of $S$, put

$$
\left(A_{\mathbf{q}}\right)_{U}:=A_{\mathbf{q}} \otimes_{A_{\mathbf{q}}\left(W_{U}\right)} \mathbf{Q}=A_{\mathbf{q}} / A_{\mathbf{q}} I_{U},
$$

where $A_{\mathbf{q}}\left(W_{U}\right)$ acts on $\mathbf{Q}$ via the symmetric character $\alpha_{U}$ and $I_{U}:=\sum_{s \in U} H_{\mathbf{q}}^{s}$ is the augmentation ideal of $A_{\mathbf{q}}\left(W_{U}\right) . A_{\mathbf{q}}^{U}$ is a right $A_{\mathbf{q}}$-module and $\left(A_{\mathbf{q}}\right)_{U}$ is a left $A_{\mathbf{q}}$-module.

We have decreasing filtrations $\left(F_{p}\right)$ and $\left(F_{p}^{\prime}\right)$ of $A_{\mathbf{q}}$, defined exactly as in (4-1) and (4-2).

If $X$ and $\mathcal{U}$ are as before, then the proof of Theorem 4.5 gives the following.

Theorem 7.1 With notation as above, for each nonnegative integer $p$,

(i) there is an isomorphism of right $A_{\mathbf{q}}$-modules:

$$
H^{*}\left(X ; \mathcal{I}\left(F_{p}\right)\right) / H^{*}\left(X ; \mathcal{I}\left(F_{p+1}\right)\right) \cong \bigoplus_{|T|=p} H^{*}\left(X, X^{S-T}\right) \otimes\left(A_{\mathbf{q}}^{T} / A_{\mathbf{q}}^{>T}\right) ;
$$

(ii) there is an isomorphism of left $A_{\mathbf{q}}$-modules:

$$
H_{*}\left(X ; \mathcal{C}\left(F_{p}^{\prime}\right)\right) / H_{*}\left(X ; \mathcal{C}\left(F_{p+1}^{\prime}\right)\right) \cong \bigoplus_{|T|=p} H_{*}\left(X, X^{T}\right) \otimes\left(H_{\mathbf{q}}^{T} / H_{\mathbf{q}}^{>T}\right)
$$

$B N$ pairs The importance of Hecke algebras lies in their relationship to buildings and $B N$ pairs (eg, see Bourbaki [1, Exercises 22 and 24, pages 56-58]). Suppose that $(G, B)$ is a $B N$ pair. Associated to $(G, B)$ we have a Coxeter system $(W, S)$ and for each $s \in S$, a subgroup $G_{s}$ of $G$ such that $G_{s}=B \cup B s B$. Put $\Phi:=G / B$. For each subset $T$ of $S$, put $G_{T}:=B W_{T} B$. Two cosets $g B$ and $g^{\prime} B$ are $s$-equivalent if they have the same image in $G / G_{s}$. This gives $\Phi$ the structure of a building. $\Phi$ has finite thickness if $\left(G_{s}: B\right)<\infty$ for all $s \in S$. If this is the case, put $q_{s}=\left(G_{s}: B\right)-1$ and regard $\mathbf{q}=\left(q_{s}\right)$ as an $I$-tuple, where $I$ is the set of conjugacy classes of elements in $S$. 
Let $F(G / B)$ denote the $\mathbf{Q}$ vector space of finitely supported, $\mathbf{Q}$-valued functions on $G / B$. The left $G$-action on $G / B$ gives $F(G / B)$ the structure of a right $G$-module. For any $T \subset S$, we have a projection $p_{T}: G / B \rightarrow G / G_{T}$. By pulling back via $p_{T}$, we can identify $F\left(G / G_{T}\right)$ with a $G$-submodule of $F(G / B)$.

Regard $F(G / B)$ as a subset of all $\mathbf{Q}$-valued functions on $G$. The Hecke algebra can be identified with the subspace of $F(G / B)$ consisting of those functions which are invariant under the $B$-action on $F(G / B)$ (induced from the left $B$-action on $G / B$ ). Under this identification, the basis element $e_{w} \in A_{\mathbf{q}}$ is identified with the characteristic function of the double coset, $C\left(w^{-1}\right):=B w^{-1} B$ and the idempotent $a_{T}$ with the characteristic function of $G_{T}\left(=B W_{T} B\right)$.

Given $f \in F(G / B)$ and $a \in A_{\mathbf{q}}$ their convolution is defined by

$$
(a * f)(h):=\int_{G} a\left(g^{-1} h\right) f(g) d g .
$$

Here we are integrating with respect to Haar measure $d g$ normalized so that the measure of $B$ is 1 . So, $A_{\mathbf{q}}$ acts from the left on $F(G / B)$ by convolution, (In fact, $A_{\mathbf{q}}$ is the intertwining algebra (= the commutant) of $G$ on $F(G / B)$.)

Lemma 7.2 For any $T \in \mathcal{S}$,

$$
A_{\mathbf{q}}^{T} \otimes_{A_{\mathbf{q}}} F(G / B)=F\left(G / G_{T}\right)
$$

Proof Let $\sum_{i} a_{T} \alpha_{i} \otimes f_{i}$ be a typical element of the left-hand side. It can be rewritten as $1 \otimes \sum a_{T} * \alpha_{i} * f_{i}$. The universal map to $F(G / B)$ consists of taking the second factor. Since $a_{T}$ is the characteristic function of $G_{T}, a_{T} * \alpha_{i} * f_{i}$ lies in $F\left(G / G_{T}\right)$; so, the image of this map is the right-hand side.

\section{Theorem 7.3}

$$
C_{c}^{i}(|\Phi| ; \mathbf{Q})=C^{i}\left(K ; \mathcal{I}\left(A_{\mathbf{q}}\right)\right) \otimes_{A_{\mathbf{q}}} F(G / B)
$$

Proof This is a direct consequence of Lemma 7.2, since

$$
C_{c}^{i}(|\Phi| ; \mathbf{Q})=\bigoplus_{c \in K^{(i)}} F\left(G / G_{S(c)}\right) .
$$

The natural conjecture is the following.

\section{Conjecture 7.4}

$$
H_{c}^{i}(|\Phi|)=H^{i}\left(K ; \mathcal{I}\left(A_{\mathbf{q}}\right)\right) \otimes_{A_{\mathbf{q}}} F(G / B) .
$$


The filtration $\left(F_{p}\right)$ induces a filtration of $H^{*}\left(K ; \mathcal{I}\left(A_{\mathbf{q}}\right)\right)$ and hence, of $H_{c}^{*}(|\Phi|)$. So, Lemma 7.2 leads us to the following.

Conjecture 7.5 In filtration degree $p$, the associated graded group of $H_{c}^{*}(|\Phi|)$ is given, as a right $G$-module, by

$$
\bigoplus_{|T|=p} H^{*}\left(K, K^{S-T}\right) \otimes F^{T} / F^{>T}
$$

where $F^{T}:=F\left(G / G_{T}\right)$ and $F^{>T}$ denotes the submodule spanned by the $F^{U}$ with $U \supsetneq T$.

\section{References}

[1] N Bourbaki, Lie groups and Lie algebras. Chapters 4-6, Elements of Mathematics (Berlin), Springer, Berlin (2002) MR1890629

[2] KS Brown, Cohomology of groups, Graduate Texts in Mathematics 87, Springer, New York (1982) MR672956

[3] M W Davis, Groups generated by reflections and aspherical manifolds not covered by Euclidean space, Ann. of Math. (2) 117 (1983) 293-324 MR690848

[4] M W Davis, The homology of a space on which a reflection group acts, Duke Math. J. 55 (1987) 97-104 MR883665

[5] M W Davis, The cohomology of a Coxeter group with group ring coefficients, Duke Math. J. 91 (1998) 297-314 MR1600586

[6] M W Davis, J Dymara, T Januszkiewicz, B Okun, Weighted L ${ }^{2}$-cohomology of Coxeter groups, preprint (2004)

[7] M W Davis, J Meier, The topology at infinity of Coxeter groups and buildings, Comment. Math. Helv. 77 (2002) 746-766 MR1949112

[8] J Dymara, T Januszkiewicz, Cohomology of buildings and their automorphism groups, Invent. Math. 150 (2002) 579-627 MR1946553

[9] A Haefliger, Extension of complexes of groups, Ann. Inst. Fourier (Grenoble) 42 (1992) 275-311 MR1162563

[10] D Kazhdan, G Lusztig, Representations of Coxeter groups and Hecke algebras, Invent. Math. 53 (1979) 165-184 MR560412

[11] L Solomon, A decomposition of the group algebra of a finite Coxeter group, J. Algebra 9 (1968) 220-239 MR0232868 
The Ohio State University, Department of Mathematics, 231 W 18th Ave Columbus, Ohio 43210-1174, USA

Instytut Matematyczny, Uniwersytet Wrocławski, pl. Grunwaldzki 2/4 50-384 Wrocław, Poland

The Ohio State University, Department of Mathematics, 231 W 18th Ave Columbus, Ohio 43210-1174, USA

University of Wisconsin-Milwaukee, Department of Mathematical Sciences PO Box 413, Milwaukee WI 53201-0413, USA

mdavis@math.ohio-state.edu, dymara@math.uni.wroc.pl, tjan@math.ohio-state.edu, okun@uwm.edu

Received: 10 April 2006 Revised: 28 June 2006 